\title{
Implementation of Mobile Apps Across Globe and Challenge to Software Engineering
}

\author{
Jung Hyun Kim1), Raja Sekhar Reddy2)
}

\begin{abstract}
The market for mobile apps is already big and it's just going to keep getting bigger. According to a recent study in 2015, 1 billion smart phones will be sold, that is twice as many as the number of personal computers right now, the average mobile app user spends more than 30 hours a month on more than two dozen apps which is a lot of time spent by a lot of people. However, there exist country differences in mobile app user behavior the users' from a particular country tend to use more apps belonging to a particular type for example users from USA are more likely to download medical apps. This paper identifies the different types of apps people from a region frequently tend to use and the challenges that the market-driven software engineering face like packaging requirements, feature space, quality expectations, app store dependency and price sensitivity.
\end{abstract}

Keywords : mobile apps, software engineering, android, portable application, smart phone.

\section{Introduction}

Progresses in versatile innovation have empowered a wide scope of utilizations to be produced that can be utilized by individuals moving[1-3]. Engineers once in a while disregard the way that clients will need to interface with such gadgets while moving. Little screen sizes, constrained availability, high power utilization rates and constrained info modalities are only a percentage of the issues that emerge when outlining for little, compact gadgets[4-5]. One of the greatest issues is the setting in which they are utilized. As these gadgets are intended to empower clients to utilize them while versatile, the sway that the utilization of these gadgets has on the versatility of the client is a basic component to the achievement or disappointment of the application[6-9]. Abb and flow examination has shown that intellectual over-burden can be an imperative part of convenience. It appears likely that cell phones may be especially

Received(March 31, 2015), Review Result(1st: April 17, 2015, 2nd: May 15, 2015), Accepted(September 10, 2015)

${ }^{1}$ Conversing Technology, Hoseo Graduate School of Venture, Seoul email: hyun2@hanmail.net

${ }^{2}$ (Corresponding Author) Vetri Vikas Educational Institutions, Mallur (Via), Rasipuram Taluk, Annamalaipatti, Tamil Nadu, India email: tavvarajasekharreddy@gmail.com 
delicate to the impacts of psychological over-burden, because of their conceivable organization in various assignment settings and confinements of size. This part of ease of use is frequently neglected in existing ease of use models, which are laid out in the following segment, as these models are intended for applications which are from time to time utilized as a part of a portable connection. The convenience model for portable applications, which we then present, fuses psychological burden as this characteristic straightforwardly affects furthermore, may be affected by the ease of use of an application. A writing audit, sketched out in the accompanying area, was directed as approval of the model. This writing audit analyzed which qualities of convenience, as characterized in the convenience model, were utilized amid the assessment of portable applications introduced in a scope of papers distributed somewhere around 2008 and 2010 a gander at the exploration strategies utilized as a part of portable $\mathrm{HCI}$, yet did not inspect the specific characteristics of ease of use fused in the model. The addition introduce the aftereffects of the writing audit.

The effect of this work on future ease of use studies and what lessons different analysts ought to consider when performing ease of use assessments on portable applications are likewise talked about. This model expands on existing speculations of ease of use yet is customized particularly for applications that can be utilized on cell phones. The ease of use model is portrayed in one next to the other with Nielsen's what's more, the meaning of ease of use. The convenience model consolidates the characteristics of both the standard what's more, Nielsen's model furthermore presents the property of psychological burden which is of specific significance to portable applications. The accompanying segment presents the ease of use model and portrays in subtle element each of the properties of convenience specified underneath as well as the three ease of use elements that are a piece of this model: client, undertaking and setting. The ease of use model for versatile applications distinguishes three elements (Client, Errand and Connection of utilization) that ought to be considered when outlining portable applications that are usable. Each of these elements will affect the last outline of the interface for the portable application. Notwithstanding this the model additionally recognizes seven qualities that can be utilized to characterize measurements to quantify the convenience of an application. The accompanying segment diagrams each of these elements and traits in more detail. The ease of use model distinguishes three elements which can influence the general ease of use of a versatile application Client, Errand and Setting of utilization. Existing ease of use models, for example, those proposed by the and Nielsen additionally perceive these variables as being basic to the fruitful ease of use of an application. For versatile applications Connection of utilization assumes a basic part as an application may be 
utilized as a part of various, altogether different settings. It is in this way fundamental for application architects to take a gander at option data techniques. A few clients may think that its hard to utilize some of these strategies because of physical confinements. For instance it has been indicated that some Tetraplegic clients who have constrained portability in their furthest points have a tendency to have high blunder rates when utilizing touch screens and this may bring about unsatisfactory troubles with certain size targets. Another component that ought to be considered is the client's past experience. In the event that a client is a specialist at the picked assignment then they are liable to support alternate way keys to perform this assignment. Then again beginner clients might lean toward an interface that is natural and simple to explore also, which permits them to find what they require. This exchange off must be considered amid the configuration of the application. Assignment The word undertaking alludes here to the objective the client is attempting to fulfill with the versatile application. Amid the advancement of utilizations, extra components can be added to an application keeping in mind the end goal to permit the client to achieve more with the product. This additional usefulness comes to the detriment of convenience as these extra components build the many-sided quality of the product and thusly the client's unique objective can get to be hard to achieve. For instance, consider a computerized camera. On the off chance that a client needs to take a photo, they should first choose between diverse modes and at that point start to line up the shot. This issue is further aggravated if the client needs to take a photo at night and needs to seek through various menu things to find and turn on an electric lamp.

In a roundabout way evaluates the traits of ease of use included inside of the ease of use model. Sometimes the studies assessed numerous qualities of ease of use and accordingly the outcomes above present both the rate and the quantity of studies in which each characteristic was considered. These concentrates frequently don't unequivocally refer to ease of use or any ease of use related criteria, thus the measurements utilized for the papers' examinations were utilized to find the ease of use traits considered. This absence of exactness is presumably because of an absence of understanding as to what constitutes ease of use and the way that the traits are not orthogonal. The three most normal traits, Viability, Proficiency and Fulfillment, relate to the properties distinguished by the standard for convenience. A reason these properties are so generally considered is their immediate relationship to the specialized capacities of the framework. Both Viability and Proficiency are identified with the outline and usage of the framework as are normally tried completely. These qualities are additionally generally simple to quantify. In most cases the Adequacy of the framework is assessed by checking whether a client can fulfill a pre-indicated assignment. Effectiveness can be measured 
by discovering the time taken by the member to finish this assignment. Surveys what's more, organized meetings can be utilized to decide the Fulfillment of clients towards the framework. mand line towards GUI based interfaces. The parts of ease of use that were viewed as minimum regularly in the papers investigated are Learnability and Memorability. There are various explanations behind this. The way of these characteristics requests that they are assessed over periods of time. To adequately measure Learnability, clients' advancement should be checked at standard interims or followed over numerous culminations of an undertaking. In the papers audited, Learnability was typically measured in a roundabout way by the changes in adequacy or productivity over numerous fruitions of a predefined errand. Memorability was just measured subjectively in the papers inspected. One approach to dispassionately measure Memorability is to look at members' utilization of the framework after a time of dormancy with the framework. The down to earth issue of selecting members who are willing to return numerous times to take an interest in an assessment is presumably a reason why this property is definitely not frequently measured dispassionately. Separates portable applications from additional conventional applications is the capacity of the client to utilize the application while moving. In this connection, the consideration is partitioned between the demonstration of moving and utilizing the application. For considering subjective burden. Some of these studies utilized the change as a part of execution of the client performing the essential undertaking as a sign of the subjective burden. Different studies utilized the to subjectively measure psychological burden.

\section{Literature review}

Existing exploration into comprehension the needs of an expansive populace of application clients and their application client conduct can be arranged into those that mine application store information, those that gather action logs from cell phones, and those that lead reviews and inspire input from clients.

The Testing in the applications of the mobiles has the many views in which they need to be performed so that the methods such as the Unit Testing, Integration Testing, Strctural Testing, Functional Testing, Performance and Reliability Testing. All these testing methodologies involve deeply in finding the application will be performed in a good manner so that no interface fault or the accessible for the customer who are using the particular mobile application on any OS platform such as the Android, Windows, IOS. 


\subsection{Backgorund Review}

Application stores have gathered a lot of information, for example, application depictions, client appraisals, and surveys. All things considered, an expanding number of studies to comprehend client needs are led by mining information from the application stores themselves gathered information on client appraisals and audits for the main 25 free and paid applications of one nation on and from each application class in the Apple iOS Application Store They utilized different factual measures to examine how and when clients give input, and in addition break down the substance of the surveys. Their outcomes demonstrated that most client surveys were given not long after new discharges, with a rapidly diminishing recurrence over the long haul. Users surveys normally contain different themes. Not with standing this Utility as the capacity of a framework to address the issues of the client. He doesn't consider this to be a piece of ease of use yet a different characteristic of a framework. In the event that an item neglects to give utility then it doesn't offer the elements and capacities required the ease of use of the item gets to be pointless as it will not permit the client to accomplish their objectives .The significant test in Portable Application Testing is the variety of cell phones with diverse capacities, components and confinements. Gadgets may have diverse specialized abilities, for example, measure of accessible memory, screen determination, screen introduction and size of the showcase, system network alternatives, support for distinctive guidelines and interface. Devices may have different technical capabilities such as amount of available memory, screen resolution, screen orientation and size of the display, network connectivity options, support for different standards and interfaces. There are numerous working frameworks that are pervasive in the versatile space like Symbian, Android, iPhone OS, Windows. Each of the working frameworks can have further adaptations for distinctive sorts of gadgets which make stage testing complex and further difficult. Another test is that the designers need to concentrate on creating applications that are anything but difficult to utilize on a portable and devour less power. The Most vital perspective that our examination, improvement furthermore, testing groups regularly miss is that portable application improvement takes significantly less time length of time then versatile application testing, contrasted with the ordinary model where application examination and advancement takes additional time priority over testing. We therefore, purposely tend give less time for testing which may bring about the application beginning to miss out to rivalry after some time. Due to this misconception and subsequently shameful testing system in versatile application; developing number of mobile. therefore, deliberately tend to give less time for testing which might result in the application starting to 
Implementation of Mobile Apps Across Globe and Challenge to Software Engineering

lose out to competition over time. Due to this misunderstanding and thus improper testing strategy in mobile application.

\subsection{Review Around}

User: Individual who communicates with the item Objective: Planned result; Connection of utilization: Clients, undertakings, gear equipment, programming and materials, and the physical and social situations in which an item is utilized. Each of the above variables may have an effect on the general configuration of the item and specifically will influence how the client will communicate with the framework. Keeping in mind the end goal to measure how usable a framework is, the ISO standard blueprints three quantifiable characteristics: Adequacy: Exactness and fulfillment with which clients accomplish determined objectives Effectiveness: Assets used in connection to the precision and fulfillment with which users uses accomplish objectives.

Fulfillment: Opportunity from distress, and positive demeanors towards the utilization of the item. Various studies concentrate on get-together prerequisites for particular applications. Distributed five amusement applications in the Android showcase and checked how the applications were utilized. Their most prevalent application gathered information from 6,907 clients. Their information demonstrated that numerous clients deserted the applications after a brief period and they recommended that engineers ought to concentrate on application quality and giving motivators to clients with a specific end goal to persuade longterm utilization of an application Hence. likewise found that the vast majority of their members were English-talking clients from the United States, thus constraining their capacity to determine determinations around a worldwide populace. Product offering testing an application on a large number of cell phones is positively an imperative test, particularly in the Android O.S., where diverse cellular telephones give distinctive elements and equipment segments, and telephone makers might profoundly redo the O.S. Considering that to the extent today there are around 130 diverse cellular telephones running Android, seven renditions of the Android OS, and accepting two firmware per gadget, this will tally to around 1800 diverse combinations the present is based on the various test on numerous gadgets practice must be supplanted by viable computerized procedures. Shared traits and variabilities utilizing a language originating from the product offering group in equipment gadgets and working frameworks may be utilized for an orderly testing of the PDA product offerings. An alternate methodology could consider to discharge instrumented beta variants of the application to be tried, make them keep running on a huge number of gadgets, and gather, store, and examine run-time information and 
disappointments. An administration gave by LessPainful.com empowers clients to transfer their applications on their site and test them on various gadgets. A short article procedures to expand scope while minimizing the quantity of gadgets utilized for testing. Testing early is dependably the right decision to add to a bug free application. The minute you are done with coding a portable application, you have to counsel the versatile application testing organization to check and alter created portable application for every conceivable issue an end client can confront. These testing organizations will give you a hand in making a bug free application that backings the most recent equipment and guarantee greatest equipment similarity. In spite of that whole portable application testing will get greatest profit for venture when there are certain surveys from clients on transferred application store. Portable application testing is tedious and costly, yet basic to guaranteeing your buyers have a positive affair when they utilize your versatile applications. It is imperative that you verify that the experience is an awesome one for each customer each time they utilize your application, beginning with the first run through. In the event that you neglect to benefit an occupation application testing, this will bring about your client doing it for you and not at all like you're trying group, your clients don't have the instruments or an ideal opportunity to report back issues. Additionally, remember that your clients would prefer not to be dealt with like guinea pigs. When they discover an imperfection, you'll never hear a word from them and they surely won't return.

Graph below gives the detailed information regarding the mobile Applications Across the Countries.

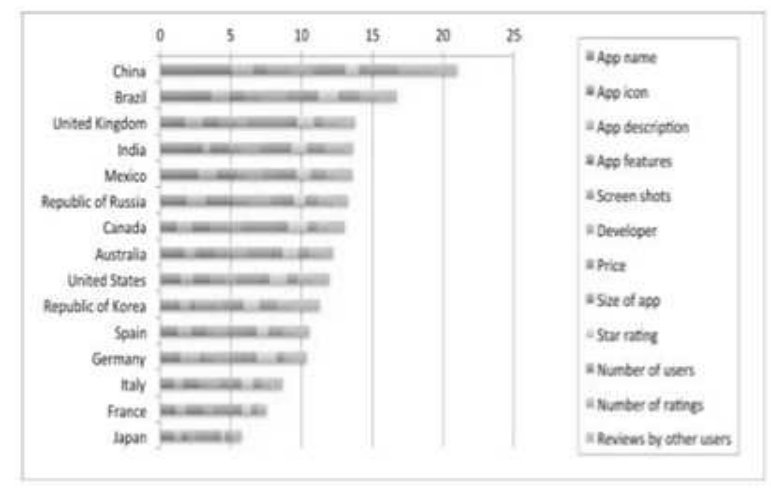

[Fig. 1] Graph representing various app behavior in different countries

\section{Theoretical analysis}




\subsection{Methodologies}

The different testing implemented have methodologies are implemented in order to test different mobile applications. For example the applications which are used in the America, Europe, Australia mainly the apps are related towards the Medical applications where the information regarding the different medicines will be shown in such mobile applications the main danger in building such apps is up to how much percentage do the reliable medicines and knowledge of the developer in the medicines so to overcome such type of the errors the testing methodologies.

\subsection{Performance and Reliable Testing}

Performance and Reliability of mobile applications will mainly depends on the mobile device resources on the content present in the application performed on the connectivity quality and variability and other contextual information are been in the performance and reliability testing and also applications such as the pay-tm applications. Run-time analysis techniques can also be adopted to monitor the resources and connectivity state and prevent performance degradation introduce a framework for analysing of the performance of contextaware mobile software systems. The experience the wide model ranges of value an environmental variable can get and model the impact of various environmental inputs into the application under test.

The people in the Europe such as the France, England, Spain, Russia, and also the people in the North America uses the applications such as the Pay-tm and many online transactions BSB services applications. For all these applications the security is the foremost thing in order to be implement the applications for the safer use of the people to download and use such type of the applications.

\subsection{Security Testing}

Security is particularly is performed for the mobility of the device to get with the operating system of the android IOS and the windows operating systems get connected to them so there chance of the thefting the information form the user mobile. So the security checking is much important in order to release the apps like that. The different security levels a defective might effect the access to personal date and the private networks and private and secured and regarding that information where the application user is located moreover the rich contextual 
information presents real privacy concerns. The mobile application publishers also increases the number of apps store the final user does not know anything about. Potentials and Automation since mobility towards different networks represent a peculiarity of mobile applications traditional security testing approaches shall be revised so to keep in consider the necessary factors that shall be simulated so to check which data is transmitted from the mobile device in the authors analyse the threats android applications pose to the security and privacy of enterprise and propose several approaches for defending enterprises against security risks.

The people who belong to the Asia continent such as the countries like India China Japan etc the people mainly concentrates on the mobile applications such as the Business applications, Entertainment apps and popular video and the audio apps. To ensure the applications provide all the services it is a heavy issue to the in order to select the various applications in the app store so the various measures are been taken into the account the developers provide the Graphical User Interface in the standard form in such a way that the user interact the and use the application so ease and comfort levels of the app need to be high.

\subsection{Different methodologies}

Mobile application languages include the constructs for managing mobility of the applications, sensing, and energy consumption. The similarities used in the new testing methodologies need have to be taken into account when producing control and the data flow graphs and their respective criteria followed in and out of the mobile business and the entertaining applications. Potentials and automation of the innovative testing technique been used in the modern testing tool, along with these other testing methodologies are also considered in to the account for the other various of testing where the rigrous testing is required to get the application is to be released correctly without fault proneness the coverage criteria and if it also too needed in order to take the new control, shall be though as a way to consider at best the new mobility, sensing, and energy constructs. In case of the new testing technique is needed the other alternative method is implemented, This is Likewise the other basic testing stratagies which are used in the windows and also the other software and typical cloud applications are been tested using the Unit testing there is need to implement the automation testing tools when compared to that of the manual testing tools which supports and enables the specification in the values and context-based areas which are typical and needed to be concentrated. IOS provides guidelines on how to perform unit testing on various tools which are been available in the market. Android enables the use of JUnit for unit testing 
android applications. However, intra- and inter-application information flow must be followed by the existing integration testing approaches have the potentials to be adapted for mobile applications at the best of our knowledge, mature research on integration testing of mobile applications is still missing today.

While a few contrasts are identified with technologic contrasts as on account of application store mindfulness in Asia the reasons for different contrasts are maybe more intricate and hard to track. The distinctions in client practices are to a great extent autonomous of Gross domestic product when positioned all together of contrasts, the rankings don't compare to the relative abundance of those nations. Our outcomes demonstrate that nation particular contrasts exist in all classifications clients from the UK are most distracted about their applications and most affected by value, clients from Japan incline toward not to rate applications, clients from China will probably choose the first application on the rundown more than whatever other clients from Mexico believe that paid applications have more components and clients from Germany and Russia will probably download reference applications. The previous gathering of clients is likewise less inclined to rate an application keeping in mind the end goal to tell others that it is great contrasted with the recent.

\subsection{Analysis}

The goal of this work is to comprehend client reception of the application store idea, their application needs, and their method of reasoning for selecting or relinquishing an application and the distinctions crosswise over nations. To accomplish the goal, we planned study inquiries to compare to each of the examination questions in For instance, we requested that members indicate the make, model name and number of the cell phone they use, and in addition the application store they utilize. We utilized close-finished inquiries at whatever point conceivable on the grounds that open-finished inquiries require a great deal more exertion from the respondents. For each shut finished inquiry, we collected a rundown of choices assembled from the writing, our past examination, and our encounters as application clients and application For instance, for client dissemination crosswise over versatile application stages, we ordered a rundown of prevalent application stages including Apple, Google Play, Blackberry, Windows Telephone. For components that impact the decision of applications, we incorporated a rundown of things the client can find in the screen of procurement, for example, application symbol, application portrayal, star appraisals, and screen shots. Past exploration has 
demonstrated that nature of the symbol impacts the client's impression of application quality and their choice of whether to download. The analysis endeavored to catch the full assortment of human conduct including those that were already obscure. In this manner we incorporated a choice where pertinent. We worded our overview precisely keeping in mind the end goal to maintain a strategic distance from any misconception of the survey. The utilized dialect that can be effortlessly comprehended by members from ages 12 or more, and utilized unambiguous words. For instance, as designer is not a typical word, we substituted it with "individual who added to the application. Specialized or phenomenal words were trailed by cases. Case in point, for the application classification Utilities we gave cases of applications fitting in with the classification, for example, Mini-computer and Wake up timer. At the point when getting some information about how every now and again clients visit the application store, we gave quantifiable choices, for example, once per day or once per month, instead of much of the time or once in a while, which are subjective words. We organized the inquiries in order to draw in the members in the overview on the grounds that members who are intrigued will probably finish the review and give better quality information. For instance, we assembled the inquiries specifically and masterminded inquiries to have a characteristic movement applications, to what impacts them while downloading applications, the sum they spend on applications, to why they rate applications, and why they quit utilizing applications. The questions toward the end in light of the fact that they are viewed as exhausting and could be understood as meddlesome toward the begin of the study. To decrease reaction inclination, we randomized the requesting of the answer decisions for decisions that don't should be sorted all together answers for the application store questions. This strategy diminishes inclination that may happen when respondents pick answers without perusing the majority of the choices. In doing as such, a few alternatives and applications remain the first choice so that members who don't do those things can rapidly proceed onward to the following inquiry, and a few choices, for example, Other remain the last choice where individuals for the most part discover them. To guarantee members don't pass up a great opportunity any inquiries, the online poll highlights missing answers and respondents can't continue until the missing answers are finished. The additionally utilized skip rationale with the goal that respondents don't see addresses that are not pertinent to them and respondents who demonstrate that they don't claim a cell phone or their cell phone can't run applications were screened out. At long last, we tried the survey on regular programs, including Web Voyager v6, Apple Safari v3, Mozilla Firefox v4 and Google Chromev2. The enrollment of the members to pre-test the poll so as to distinguish potential issue. Choosing the members to reflect, however much as could reasonably be expected, the 
fluctuated demographics of our intended interest group regarding age and nations like the United Kingdom, Germany, Japan, China, and Australia countries. Requested that the members finish the poll and call attention to any issues they experienced. Specifically, we asked them to

(1) Highlight uncertain directions, inquiries, and choices.

(2) Recognize missing inquiries and choices to the study inquiries.

(3) Point out changes we can make to the poll so as to spur potential respondents.

\section{Experimental investigation}

\subsection{Laboratory Analysis}

There are a few points of interest of performing ease of use testing of portable applications through controlled research center analyses. Initial, an analyzer has full control over an test.The user can characterize specific errands and strategies that match the objective of a ease of use study, and guarantee that members take after trial directions. For case if the target of a study is to research the adequacy of an information passage strategy while a client is moving around, then a research center investigation is more suitable than a field study on the grounds that analyzers can unequivocally require and guarantee members to utilize a cell phone while moving. Second, it is anything but difficult to quantify convenience traits and decipher results through controlling other unessential variables in a environment. Accordingly the research facility test methodology is exceptionally useful to convenience thinks about that emphasis on looking at various interface outlines or information data instruments for cell phones. Third, it makes it conceivable to utilize video or sound recording to catch members' response counting feelings when utilizing an application A noteworthy constraint of the research center testing strategy is that it overlooks versatile setting and inconsistent association of remote systems. A portable application tried in a genuine environment may not function and also it does in a controlled research facility setting because of the changing and flighty system conditions and other ecological variables. In a lab, members may not encounter the potential unfriendly impacts of those relevant components. Field concentrates on. A noteworthy point of preference of leading ease of use tests through field studies is that it takes dynamic portable connection and inconsistent remote systems into thought, which are hard to mimic in research center examinations. The apparent ease of use of a portable Application is inferred in view of members' involvement in a genuine domain, which is possibly more solid and practical contrasted with research facility Not performing field considers for portable applications is a 
long way from trifling. A noteworthy. Test of this approach lies in the absence of adequate control over members in a study. There are three principal troubles reported in the writing. To start with, it can be confounded to set up practical situations that catch the abundance of the portable setting. Second, it is difficult to apply settled assessment systems, for example, perception and verbal convention when a test is directed in a field. Third, on the grounds that clients will physically move around in a progressively evolving environment, it is trying for information gathering and condition control. Hence, in a field study, analyzers must characterize the extent of portable.

The below diagram represents detailed analysis of the Mobile Application Testing on the Various Fields required for the various applications in the different countries across the world.

\subsection{Field Studies}

The cell phones utilized by customers make the most clear test to versatile testing. Possibly a huge number of distinctive customer gadgets could be utilized to get to your portable application or site, and they should subsequently all be considered when testing your versatile applications. Add to this the diverse adaptations of working frameworks, and the stages get insane enormous! You can give up scope crosswise over gadget/OS mixes to a degree, however when you decrease the quantity of gadget sorts that you test against, you are taking a risk that your application won't not work for various potential clients. To handle the gadget move, you have three alternatives: You can test solely utilizing genuine gadgets, you can test only with copied gadgets, or you can utilize a blend of both.

Genuine gadgets have the benefit of having the greater part of the confinements and characteristics present in the real customer working framework, equipment, and firmware blend utilized by your objective buyers. Notwithstanding, testing with genuine gadgets can jkupon to deal with themselves and their prompt families just.

1. Manliness List manly social orders have unmistakably particular enthusiastic sex parts: men should be emphatic, extreme, and concentrated on material achievement, while ladies should be more unassuming, delicate, and worried with the personal satisfaction.

2. Instability Evasion File the extent to which the individuals from a general public feel uncomfortable with vulnerability and equivocalness.

3. Long haul Introduction Record the cultivating of ideals situated towards future, for example, perseverance and individual flexibility.

4. Liberality Versus Limitation Record liberal social orders tend to permit generally free 
satisfaction of fundamental and characteristic human yearnings identified with appreciating life and having some good times, controlled social orders have a conviction that such delight should be checked and managed by strict norms.

\subsection{Country differences}

While some differences are related to technologic differences as in the case of app store awareness in Asia the causes of other differences are perhaps more complex and difficult to track. The differences in user behaviours are largely independent of GDP when ranked in order of differences. The former group of users is also less likely to rate an app in order to let others know that it is good compared to the latter. In previous work, individualist cultures are less likely to share information with their groups in individualist countries, media is primary source of information. In collectivist countries, social network is primary source of information. One universal factor worldwide is app abandonment - all users are very likely to cease using apps of bad quality for example crashes too slow difficult to use does not work It seems that only an effectively engineered app will stand the test of time and become a popular addition to the mobile device of users. Evidence for this can also be seen in the participants responses when asked to name of the app they spent most money on and describe the best and worst feature of the app. One of the most common answers was with very positive feedback on simplicity and ease of use. The Graph below gives information regarding comparision of serval features.

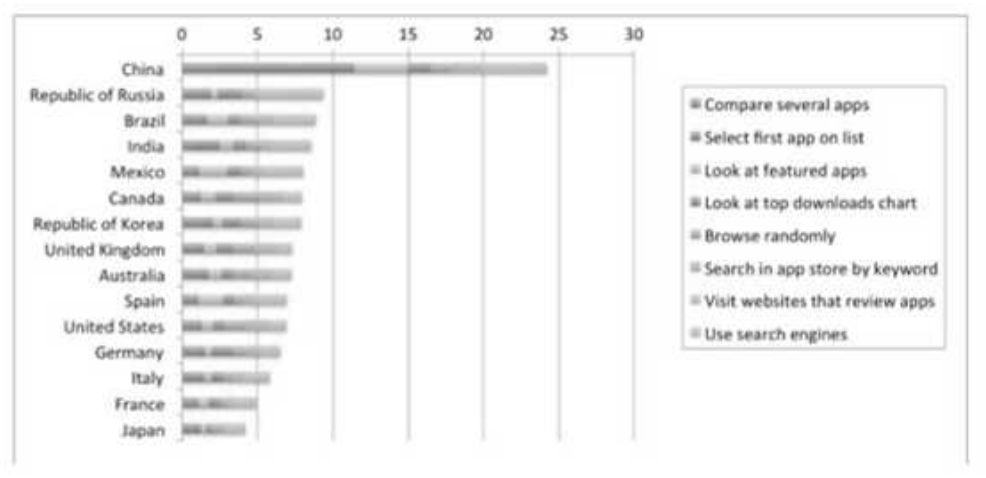

[Fig. 2] Variation of features in different Countries 


\section{Conclusion}

Portable applications are programming created for use on cell phones and made accessible through application stores. Application stores are exceedingly aggressive markets with a quickly expanding number of applications, and designers need to take into account a substantial number of clients because of low edges per deal. In this study, we led one of the biggest reviews to date of portable application clients over the world. We exhibited that application client conduct varies altogether crosswise over nations, an outcome that was appeared in different areas yet at no other time in application based programming designing, demonstrating that application engineers ought to deliberately consider the nations of their objective clients. We additionally examined client appropriation of the application store idea, their application needs, and their basis for selecting or forsaking an application. Through investigation of the review results, we distinguished new difficulties to market-driven programming building identified with bundling necessities, highlight space, quality desires, application store reliance, value affectability, and biological system impact, and their suggestions for programming designing examination as far as exploration headings and apparatus improvement.

Discharged the consequences of our study to the application engineer group and got criticism that the bits of knowledge are exceptionally valuable. A few engineers have asked for different nations to be concentrated on as they are building applications for those nations. suspect that the new difficulties distinguished in this paper can control programming building specialists towards the advancement of instruments and systems to enhance business sector driven programming designing for versatile applications.

\section{References}

[1] J. N. Dag, Elicitation and Management of User Requirements in Market-Driven Software Development, Department of Communication Systems Lund Institute of Technology, Licentiate Thesis, (2002).

[2] J. Bergman, J. Kauko, and J. Keränen, Hands on music: physical approach to interaction with digital music, Proceedings of the 11th International Conference on Human-Computer Interaction with Mobile Devices and Services, (2009), ACM; Bonn, Germany.

[3] L. Karlsson, Å. G. Dahlstedt, B. Regnell, J. N. Dag, and A. Persson, Requirements engineering challenges in market-driven software development - An interview study with practitioners, Information and Software Technology, (2007), Vol.49, No.6, pp.588-604.

[4] K. Wnuk, B. Regnell, and B. Berenbach, Scaling Up Requirements Engineering - Exploring the Challenges 
of Increasing Size and Complexity in Market-Driven Software Development, Lecture Notes in Computer Science, (2011), Vol.6606. Springer; Berlin, Heidelberg

[5] M. Bohmer, B. Hecht, J. Schoning, A. Kruger, and G. Bauer, Falling asleep with angry birds, facebook and kindle: a large scale study on mobile application usage, Proceedings of the 13th International Conference on Human Computer Interaction with Mobile Devices and Services, (2011).

[6] O. I. Franko and T. F. Tirrell, Smartphone app use among medical providers in ACGME training programs, Journal of Medical Systems, (2011), Vol.36, No.5, pp.3135-3139.

[7] B. Westerman, How do people really use the iPhone, Report. Create with Context, (2008).

[8] P. Gilbert, B. G. Chun, L. P. Cox, and J. Jung, Vision: Automated Security Validation of Mobile Apps at App Markets, Proceedings of MCS'11, (2011) June 28; Bethesda, Maryland, USA.

[9] C. L. Ventola, Mobile Devices and Apps for Health Care Professionals: Uses and Benefits, P\&T, (2014), Vol.39, No.5, pp.356-364. 